\title{
Non-linear error correction, asymmetric adjustment and cointegration
}

\author{
Alvaro Escribano ${ }^{\mathrm{a}, *}$, Gerard A. Pfann ${ }^{\mathrm{b}}$ \\ ${ }^{\mathrm{a}}$ Department of Statistics and Econometrics, Universidad Carlos III de Madrid, Calle Madrid 126, \\ Getafe, Madrid 28903, Spain \\ ${ }^{\mathrm{b}}$ Department of Quantitative Economics, Maastricht University, PO Box 616, 6200 MD \\ Maastricht, The Netherlands
}

\begin{abstract}
This article links the intertemporal choice model with the non-linear error correction (NEC) model. It has three main components. First, it outlines a model of non-linear error correction, in which the linear error correction term $\alpha^{\prime} X_{t}$ (the vector time series $X_{t}$ is cointegrated, $\alpha$ is the cointegrating vector) is replaced by the non-linear term $g\left(\alpha^{\prime} X_{t}\right)$, where $g($.$) is a non-linear function. Second, several types of asymmetries and the existence$ of multiple equilibria are discussed. The implications for the NEC model of trending targets are also explained. Third, it is shown that non-linear error correction is present in a trivariate series of UK employment, wage and capital stock. (c) 1998 Elsevier Science B.V.
\end{abstract}

JEL Classification: $\mathrm{C} 00$

Keywords: Non-linear error correction; Asymmetric adjustment; Multiple equilibria; Intertemporal optimization

\section{Introduction}

Non-linear error correction basically refers to non-linear adjustment to longrun equilibrium economic relationships. In this article we show that the concept of

\footnotetext{
* Corresponding author.
} 
non-linear error correction (NEC) model (Escribano, 1986, 1987, 1996; Granger and Lee, 1989; Escribano and Mira, 1997) has its structural counterpart in the form of optimizing a decision process under uncertainty over an infinite horizon where the decision variable is quasi-fixed and bears asymmetric costs of adjustment (Pfann and Palm, 1993; Pfann, 1996). The concept of asymmetry implies that the costs of adjusting to a higher target level are not necessarily marginally equivalent to the costs of adjusting to a lower target level.

This article has three main components. First, it outlines a model of non-linear error correction (NEC), in which the linear error correction term $\left(\alpha^{\prime} X_{t}\right.$ (the vector time series $X_{t}$ is cointegrated, $\alpha$ is the cointegrating vector) is replaced by the non-linear term $g\left(\alpha^{\prime} X_{t}\right)$, where $g($.$) is a non-linear function. Second, several types$ of asymmetries are discussed. The implications for the NEC model of trending targets are explained. Third, it is shown that non-linear error correction is present in a trivariate series of UK employment, wage and capital stock.

The article is organized as follows. In Section 2 the non-linear error correction representation is derived from a general non-linear autoregressive distributed lag model and issues of integration and cointegration (Engle and Granger, 1987) are passed in review. Section 3 presents the linear partial adjustment model. In Section 4 the characteristics of the asymmetric adjustment model are linked with the concept of non-linear error correction. In Section 5 the implications of variables having trends in mean with respect to NEC models are discussed. Section 6 presents several specifications of asymmetries in the NEC model, that can be found in the literature. It is shown that these non-linear error corrections are special cases of the general formulation presented in the article. An empirical application is given in Section 7 where the non-linear relationship is investigated between UK time series data on employment real wage costs and the stock of capital goods. Finally, in Section 8 conclusions are drawn.

\section{Non-linear error correction and cointegration}

Let $X_{t}$ be an $(N \times 1)$-vector of economic variables and suppose that we have $T$ observations of each individual series of $X_{t}$. Let $E\left(X_{t}\right)=\mu_{t}$ be an $(N \times 1)$-vector whose element can be constant terms, deterministic trends, etc. and define $\bar{X}_{t}=X_{t}$ $-\mu_{t}$. If we decompose $\bar{X}_{t}=\left(\bar{Q}_{t}, \bar{P}_{t}\right)^{\prime}$, where $\bar{Q}_{t}$ is one dimensional and $\bar{P}_{t}$ is an [(N-1) $\times 1$-vector, we can factorize the joint density of $\bar{X}_{t}$ into the conditional and the marginal (see for example Engle et al., 1983),

$$
\begin{aligned}
& D\left(\bar{X}_{t} \mid \bar{X}_{t-1}, \bar{X}_{t-2}, \ldots, \bar{X}_{0}, \theta\right) \\
& \quad=D\left(\bar{Q}_{t} \mid \bar{P}_{t}, \bar{X}_{t-1}, \bar{X}_{t-2}, \ldots, \bar{X}_{0}, \theta_{1}\right) D\left(\bar{P}_{t} \mid \bar{X}_{t-1}, \bar{X}_{t-2}, \ldots, \bar{X}_{0}, \theta_{2}\right)
\end{aligned}
$$

If the parameters of interest $\psi$ are a function of the parameters $\theta_{1}, \psi=f\left(\theta_{1}\right)$ and if $\bar{P}_{t}$ is weakly exogenous for the parameter of interest $\psi$, we can make 
inference on $\psi$ based on the conditional density without any loss of relevant information. In particular we will be interested in the conditional expectation $E\left(\bar{Q}_{t} \mid \bar{P}_{t}, \bar{X}_{t-1}, \bar{X}_{t-2}, \ldots, \bar{X}_{0}, \theta_{1}\right)$.

Let $\epsilon_{t}=\bar{Q}_{t}-E\left(\bar{Q}_{t} \mid \bar{P}_{t} \bar{X}_{t-1}, \ldots, \bar{X}_{0}, \theta_{1}\right)$ so that $\epsilon_{t}$ is a martingale difference sequence relative to the $\gamma$-algebra generated by $\left(\bar{P}_{t}, \bar{X}_{t-1}, \bar{X}_{0}\right)$. For simplicity we will assume that $\epsilon_{t}$ has a constant variance equal to $\sigma_{\epsilon}^{2}$. Suppose the conditional expectation is represented by a finite autoregressive distributed lag model with a non-linear term (see Escribano, 1987, 1996),

$$
\begin{aligned}
& E\left(\bar{Q}_{t} \mid \bar{P}_{t}, \bar{X}_{t-1}, \bar{X}_{t-2}, \ldots, \bar{X}_{0}, \theta_{1}\right) \\
& \quad-\phi^{1}(B) \bar{Q}_{t-1}-\theta(B) P_{t}-g\left(\bar{Q}_{t-1}-\alpha \bar{P}_{t-1}\right) .
\end{aligned}
$$

Then we can write the equation for $\bar{Q}_{t}$ as

$$
\phi(B) \bar{Q}_{t}+\theta(B) \bar{P}_{t}=-g\left(\bar{Q}_{t-1}-\alpha \bar{P}_{t-1}\right)+\epsilon_{t},
$$

where $\phi(B)$ is a finite lag polynomial in the lag operator $B$, with $\phi(0)=1, \theta(B)$ is a $[1 \times(N-1)]$-vector of finite polynomials in the lag operator $B$. The lag operator $B$ is such that $B^{k} \bar{X}_{t}=\bar{X}_{t-k}$ and $\theta(0)$ is a [1 $\left.\times(N-1)\right]$-vector whose elements are not all equal to 0 so that in Eq. (2.1) there are some contemporaneous weakly exogenous variables. The non-linear function $g$ is such that $-2<d g(Z) / d z<0$ (see Escribano, 1996 for details).

If both $\phi(B)$ and $\theta(B)$ have a unit root then $\bar{Q}_{t}$ and $\bar{P}_{t}$ are integrated of order one, I(1). In this case we can obtain different, but observationally equivalent, representations from Eq. (2.2). Taking Taylor series expansions, of order larger than the maximum lag of $\phi(B)$ and $\theta(B)$, around the point $B=1$ we get

$$
\phi(B)=\phi(1)=\phi^{*}(B)(1-B)
$$

and

$$
\theta(B)=\theta(1)+\theta^{*}(B)(1-B)
$$

where $\phi^{*}(B)$ and $\theta^{*}(B)$ have all roots outside the unit circle. Substituting (2.3) and (2.4) in Eq. (2.2) and rearranging terms we obtain

$$
\begin{aligned}
& \phi(1) \bar{Q}_{t}+\theta(1) \bar{P}_{t} \\
& \quad=-\phi^{*}(B)(1-B) \bar{Q}_{t}-\theta^{*}(B)(1-B) \bar{P}_{t}-g\left(\bar{Q}_{t-1}-\alpha \bar{P}_{t-1}\right)+\epsilon_{t} .
\end{aligned}
$$

Now decompose $\phi(1)=\Gamma_{1} \alpha_{1}$ and $\theta(1)=\Gamma_{1} \alpha_{2}$ and dividing (2.5) by the scalar $\Gamma_{1} \alpha_{1}$, we normalize (2.5) as

$$
\begin{aligned}
\bar{Q}_{t}= & \alpha \bar{P}_{t}-\phi^{-1}(1) \phi^{*}(B)(1-B) \bar{Q}_{t}-\phi^{-1}(1) \theta^{*}(B)(1-B) \bar{P}_{t} \\
& -\phi^{-1}(1) g\left(\bar{Q}_{t-1}-\alpha \bar{P}_{t-1}\right)+\phi^{-1}(1) \epsilon_{t},
\end{aligned}
$$


which is a non-linear version of Bewley's representation (Bewley, 1979), with $\alpha=-\phi^{-1}(1) \theta(1)$. Note that Bewley's linear representation is obtained from (2.6) by setting $g\left(\bar{Q}_{t-1}-\alpha \bar{P}_{t-1}\right)=0$. See Hylleberg and Mizon (1989) for an alternative procedure to derive this representation.

If we add and subtract $\phi(1) B$ and $\theta(1) B$ to (2.3) and (2.4), respectively, we can rewrite $\phi(B)$ and $\theta(B)$ as follows

$$
\begin{aligned}
\phi(B) & =\phi(1) B+\left[\phi^{*}(B)+\phi(1)\right](1-B) \\
& =\phi(1) B+\phi^{* *}(B)(1-B)
\end{aligned}
$$

and

$$
\begin{aligned}
\theta(B) & =\theta(1) B+\left[\theta^{*}(B)+\theta(1)\right](1-B) \\
& =\theta(1) B+\theta^{* *}(B)(1-B),
\end{aligned}
$$

Substituting (2.7) and (2.8) into (2.2) we obtain a non-linear error correction representation

$$
\begin{aligned}
\phi^{* *} & (B)(1-B) \bar{Q}_{t}+\theta^{* *}(B) \bar{P}_{t} \\
& =-\phi(1) \bar{Q}_{t-1}-\theta(1) \bar{P}_{t-1}-g\left(\bar{Q}_{t-1}-\alpha \bar{P}_{t-1}\right)+\epsilon_{t} .
\end{aligned}
$$

Decomposing the long-term components as $\phi(1)=\Gamma_{1} \alpha_{1}, \theta(1)=\Gamma_{1} \alpha_{2}$ and dividing by the scalar $\alpha_{1}$ we can normalize (2.9) getting a more explicit representation

$$
\begin{aligned}
& \phi_{\alpha}(B)(1-B) \bar{Q}_{t}+\theta_{\alpha}(B)(1-B) \bar{P}_{t} \\
& \quad=-\Gamma_{1}\left(\bar{Q}_{t-1}-\alpha \bar{P}_{t-1}\right)-g_{\alpha}\left(\bar{Q}_{t-1}-\alpha \bar{P}_{t-1}\right)+\epsilon_{\alpha t} .
\end{aligned}
$$

where $\phi_{\alpha}(B)=\left(1 / \alpha_{1}\right) \phi^{* *}(B), \theta_{\alpha}(B)=\left(1 / \alpha_{1}\right) \theta^{* *}(B), g_{\alpha}()=.\left(1 / \alpha_{1}\right) g($.$) and$ $\epsilon_{\alpha t}=\left(1 / \alpha_{1}\right) \epsilon_{t}$. If $g_{\alpha}()=$.0 , we obtain the linear error correction model.

In general the function $g_{\alpha}\left(\bar{Q}_{t-1}-\alpha \bar{P}_{t-1}\right)$ incorporates all departures from the linear and symmetric error correction term, $\Gamma_{1}\left(\bar{Q}_{t-1}-\alpha \bar{P}_{t-1}\right)$. For models (2.6) and (2.10) to be balanced, $\bar{Q}_{t}-\alpha \bar{P}_{t}$ must be I(0) since $\bar{Q}_{t}$ and $\bar{P}_{t}$ are both I(1), with $(1,-\alpha)^{\prime}$ being the cointegrating vector. Also it must hold that a non-linear function $g \alpha[\mathrm{I}(0)]$ is still $\mathrm{I}(0)$. This last condition is generally satisfied under $\alpha$-mixing or near epoch dependence conditions (see Escribano, 1987; Escribano and Mira, 1997).

The error correction and Bewley's representations are observationally equivalent although in practice one can be preferred over the other. Wickens and Breusch (1988) mentioned that Bewley's representation has the advantage of giving the correct standard errors for the longrun coefficients at the costs of requiring instrumental variables estimation (IV), since the error term $\epsilon_{t}$ is correlated with the regressor $(1-B) \bar{Q}_{t}$. On the other hand, the error correction representation can be estimated by OLS and the standard errors of the longrun coefficients may 
be obtained after some calculations (Banerjee et al., 1993) or by non-linear least squares (Stock, 1987; Escribano and Mira, 1997).

\section{The linear partial adjustment model}

In the linear partial adjustment model a representative economic agent is assumed to construct a contingency plan at time $t$ for a purely non-deterministic quasi-fixed decision variable ${ }^{1} Q$ in order to minimize the expected real present value of a quadratic loss-function over an infinite time horizon. The optimization problem is as follows

$$
\underset{Q}{\operatorname{Minimize}} E\left[\sum_{i=0}^{\infty} \beta^{i}\left(\left(Q_{t+i}-Q_{t+i}^{*}\right)^{2}+\gamma\left((1-B) Q_{t+i}\right)^{2}\right) \mid \Omega_{t}\right],
$$

where $E$ is the mathematical expectations operator, $\Omega_{t}$ is the conditioning set of available information at time $t, \beta$ is a real discount value lying between zero and one, $\gamma$ is a constant positive parameter measuring the adjustment costs of changing the level of $Q$ over time. $Q^{*}$ is the target level of $Q$ and is assumed to be linearly related to the firms purely non-deterministic forcing variables $P_{t}$ and a stochastic zero mean shock $u_{t}$

$$
Q_{t}^{*}=\alpha P_{t}-u_{t}
$$

where $\alpha^{\prime}$ is a $[(N-1) \times 1)$-vector of constant parameters. If $Q_{t}=Q_{t}^{*}$, Eq. (3.2) can be interpreted as the longrun equilibrium relation between $Q$ and $P$, also known as the cointegration relationship, with $(1,-\alpha)$ being the cointegration vector.

The first order condition for (3.1) at time $t$ is

$$
Q_{t}+\gamma(1-B) Q_{t}-\beta \gamma E\left[(1-B) Q_{t+1} \mid \Omega_{t}\right]=Q_{t}^{*}
$$

or

$$
E\left[(1-B) Q_{t+1} \mid \Omega_{t}\right]=\beta^{-1}(1-B) Q_{t}+(\beta \gamma)^{-1}\left(Q_{t}-Q_{t}^{*}\right) .
$$

The left-hand side of (3.4) cannot be observed as such, but the forward looking closed form solution for the inhomogeneous second order linear difference equation is wellknown in literature and can be written as the partial adjustment representation (see Nickell, 1985)

$$
(1-\lambda B) Q_{t}=(1-\lambda)(1-\beta \lambda) \sum_{i=0}^{\infty}(\beta \lambda)^{i} E\left[Q_{t+i}^{*} \mid \Omega_{t}\right]
$$

\footnotetext{
${ }^{1}$ In Section 5 we generalize this approach to the case of variables having trends in the means.
} 
where $\lambda$ is the root of the characteristic equation

$$
\lambda^{2}-\left(1+\beta^{-1}+(\beta \gamma)^{-1}\right) \lambda+\beta^{-1}=0,
$$

that lies within the unit circle, being

$$
\lambda=\frac{1}{2}\left(1+\beta^{-1}+(\beta \gamma)^{-1}\right)-\frac{1}{2}\left(\left(1+\beta^{-1}+(\beta \gamma)^{-1}\right)^{2}-4 \beta^{-1}\right)^{1 / 2}
$$

since both roots are real and lie on either side of the unit circle (see Palm and Pfann, 1991). Without loss of generality we may assume that the generating process of $P_{t}$ is an autoregressive process, where $T(B)$ is the corresponding autoregressive lag polynomial. Then we substitute (3.2) into (3.5) and obtain (see Hansen and Sargent, 1980)

$$
\begin{aligned}
(1-\lambda B) Q_{t}= & \alpha(1-\lambda)(1-\beta \lambda)\left(T(\beta \lambda)\left(1-\beta \lambda B^{-1}\right)\right)^{-1}(T(\beta \lambda) \\
& \left.-\beta \lambda B^{-1} T(B)\right) P_{t}+(1-\lambda)(1-\beta \lambda) u_{t}
\end{aligned}
$$

which may be simplified into the unique closed form solution of (3.1)

$$
Q_{t}=\lambda Q_{t-1}+\alpha(1-\lambda) T^{*}(B) P_{t}+(1-\lambda)(1-\beta \lambda) u_{t}
$$

where $T^{*}(B)=(1-\beta \lambda)\left(T(\beta \lambda)\left(1-\beta \lambda B^{-1}\right)\right)^{-1}\left(T(\beta \lambda)-\beta \lambda B^{-1} T(B)\right)$.

$T^{*}(B) P_{t}$ is known as the forward looking target of the linear partial adjustment model. The zero mean process of stochastic shocks $u_{t}$ is predominantly found to follow an autoregressive process in the empirical literature on flexible adjustment mechanisms. The resulting autocorrelation in the residual error of (3.9) is eliminated applying the Koyck transformation procedure, transforming (3.9) into

$$
(1-\lambda B) \phi(B) Q_{t}=\alpha(1-\lambda) \theta(B) P_{t}+\eta_{t}
$$

where $\eta_{t}$ is a white noise innovation. If, in accordance with Section 2, $Q_{t}$ as well as $P_{t}$ have unit roots the partial adjustment model (3.10) can be written as a linear error correction model with $g_{\alpha}()=$.0 (see also Nickell, 1985)

$$
\phi_{\alpha}(B)(1-B) Q_{t}=-(1-\lambda)\left(Q_{t-1}-\alpha P_{t-1}\right)+\alpha(1-\lambda) \theta_{\alpha}(B)(1-B) P_{t}+\eta_{t}
$$

where $\phi_{\alpha}(B)$ and $\theta_{\alpha}(B)$ have all roots outside the unit circle.

Define $\phi_{\lambda}(B)=(1-\lambda B) \phi(B)$. Now we can write (3.10) as

$$
\phi_{\lambda}(B) Q_{t}=\alpha(1-\lambda) \theta(B) P_{t}+\eta_{t} .
$$

Decomposing the polynomials $\phi_{\lambda}(B)$ and $\theta(B)$ according to Eq. (2.3) and Eq. (2.4) we get 


$$
\begin{gathered}
\phi_{\lambda}(1) Q_{t}-\alpha(1-\lambda) \theta(1) P_{t}=-\phi_{\lambda}^{*}(B)(1-B) Q_{t} \\
+\alpha(1-\lambda) \theta^{*}(B)(1-B) P_{t}+\eta_{t} .
\end{gathered}
$$

Dividing Eq. (3.13) by $\phi_{\lambda}(1)$, we obtain Bewley's representation

$$
\begin{aligned}
Q_{t}= & \alpha^{*} P_{t}-\phi_{\lambda}(1)^{-1} \phi_{\lambda}^{*}(B)(1-B) Q_{t} \\
& +\phi_{\lambda}^{-1} \alpha(1-\lambda) \theta^{*}(B)(1-B) P_{t}+\phi_{\lambda}^{-1}(1) \eta_{t}
\end{aligned}
$$

where $\alpha^{*}=\phi_{\lambda}^{-1}(1) \alpha(1-\lambda)$.

\section{Asymmetric adjustment and non-linear error correction}

In this section we implement the asymmetric adjustment costs flexible functional form proposed by Pfann and Verspagen (1989) into the structural partial adjustment model. The economic agent chooses a contingency plan at time $\mathrm{t}$ for a quasi-fixed decision variable $Q$ in order to minimize the expected real present value of a non-linear loss-function over an infinite time horizon. The optimization problem with asymmetric adjustment costs (AAC) is as follows

$$
\underset{Q}{\operatorname{Min}} E\left\{\sum_{i=0}^{\infty} \beta^{i}\left(\left(Q_{i}-Q_{t-i}^{*}\right)+\operatorname{AAC}\left((1-B) Q_{t+i}\right) \mid \Omega_{t}\right\}\right.
$$

with

$$
\begin{aligned}
\operatorname{AAC}\left((1-B) Q_{t}\right)= & \gamma\left((1-B) Q_{t}\right)^{2}+2\left(\exp \left(\delta(1-B) Q_{t}\right)\right. \\
& -\left(1+\delta\left((1-B) Q_{t}\right)\right) .
\end{aligned}
$$

The constant parameter $\delta$ measures the difference in costs between an increase in $Q$ and a decrease in $Q$. If $\delta>0$, marginal costs of increasing $Q$ exceed costs of reducing $Q$. If $\delta<0$, marginal costs of reducing $Q$ exceed costs of increasing $Q$. If $\delta=0,(4.1)$ is just the linear-quadratic optimization problem discussed in the previous section.

Hence, the symmetric linear partial adjustment model is nested in the asymmetric model (4.1). We note that the asymmetric specification is strictly convex under the standard assumption of $\gamma$ being positive. The exponential AAC also encompasses polynomial approximations of many non-linear functions.

The first order necessary conditions for (4.1) are as follows

$$
\begin{aligned}
& \beta E\left\{\gamma(1-B) Q_{t+1}+\delta\left(\exp \left(\delta(1-B) Q_{t+1}\right)-1\right) \mid \Omega_{t}\right\} \\
& \quad=\gamma(1-B) Q_{t}+\left(Q_{t}-Q_{t}^{*}\right)+\delta\left(\exp \left(\delta(1-B) Q_{t}\right)-1\right) .
\end{aligned}
$$

If $(1-B) Q_{t}$ and $Q_{t}-Q_{t}^{*}$ are stationary, the structural parameters of the Euler Eq. (4.3) can be estimated consistently using GMM. This approach has been 
followed in Pfann and Palm (1993) and Pfann (1996), for example. The approach of Hamilton (1989) is to transform the data into discrete Markov processes, arguing that non-linearities in the data are generated by stochastic processes that are subject to discrete shifts in regime. We believe that valuable information being present in the data will be lost using Hamilton's transformation method. Novales (1990) proposed a solution technique for non-linear models positting stochastic processes for the decision variable $Q_{t}$ in order to solve the model for the forcing variables. This method is untractable with respect to our approach, since the parameters of asymmetry have to be chosen a priori in Novales' method. Palm and Pfann (1997) solve (4.3) numerically using a parameterized expectations algorithm.

Yet, a suitable approximation of the closed form solution may exist and using additional information more efficient estimates of the structural parameters may be obtained. Granger and Lee (1989) considered error correction models where the positive residual error of the longrun relationship, $\max \left(Q_{t-1}-\alpha P_{t-1} ; 0\right)$ and the negative residual error of the longrun relationship, $\min \left(Q_{t-1}-\alpha P_{t-1} ; 0\right)$, have been introduced into the model as separate regressors.

The optimization model with asymmetries in adjustment costs (4.1) is the structural counterpart of the asymmetric error correction model. To measure the asymmetric error correction we introduce the following concepts.

Positive error correction movements are characterized by positive differences between two subsequent measurement points of the longrun equilibrium error

$$
\left(Q_{t}-\alpha P_{t}\right)^{+} \equiv\left[\begin{array}{ll}
\left(Q_{t}-\alpha P_{t}\right) & \text { iff }(1-B)\left(Q_{t}-\alpha P_{t}\right)>0 \\
& 0 \text { otherwise }
\end{array}\right.
$$

Negative error correction movements are characterized by negative differences between two subsequent measurement points of the longrun equilibrium error

$$
\left(Q_{t}-\alpha P_{t}\right)^{-} \equiv\left[\begin{array}{ll}
\left(Q_{t}-\alpha P_{t}\right) & \text { iff }(1-B)\left(Q_{t}-\alpha P_{t}\right)<0 \\
& 0 \text { otherwise }
\end{array}\right.
$$

The non-linear function $g \alpha($.) introduced in (2.10) is hence expressed as follows (see Fig. 2b where $\left.Q_{t}-\alpha P_{t}=u_{t}\right)^{2}$

$$
\begin{aligned}
& \Gamma_{1}\left(Q_{t}-\alpha P_{t}\right)+g_{\alpha}\left(Q_{t}-\alpha P_{t}\right) \\
& \quad=-\left(1-\lambda_{1}\right)\left(Q_{t}-\alpha P_{t}\right)^{-}-\left(1-\lambda_{2}\right)\left(Q_{t}-\alpha P_{t}\right)^{+},
\end{aligned}
$$

since $(1-B)\left(Q_{t}-\alpha P_{t}\right)=\left(Q_{t}-\alpha P_{t}\right)^{+}+\left(Q_{t}-\alpha P_{t}\right)^{-}$, whereas $\lambda_{1}$ and $\lambda_{2}$ correspond with $\lambda$ in Eq. (3.11) such that $\frac{1}{2}\left(\lambda_{1}+\lambda_{2}\right)=\lambda$.

The corresponding asymmetric error correction model can be obtained substituting (4.5) for $-(1-\lambda)\left(Q_{t-1}-\alpha P_{t-1}\right)$ in (3.11). This gives

\footnotetext{
${ }^{2}$ More general non-linear adjustments will be considered in Section 6 .
} 


$$
\begin{aligned}
& \phi_{\alpha}(B)(1-B) Q_{t}=-1\left(1-\lambda_{1}\right)\left(Q_{t-1}-\alpha P_{t-1}\right)^{-} \\
& \quad-\left(1-\lambda_{2}\right)\left(Q_{t-1}-\alpha P_{t-1}\right)^{+}+\alpha(1-\lambda) \theta_{\alpha}(b)(1-B) P_{t}+\eta_{t} .
\end{aligned}
$$

Eq. (4.6) can be analyzed using a two step estimation technique, as proposed by Engle and Granger (1987). First, one estimates the cointegrating vector $\alpha$ by OLS, $\hat{\alpha}$. Second, Eq. (4.6) can be estimated with $\left(Q_{t-1}-\hat{\alpha} P_{t-1}\right)^{-}$and $\left(Q_{t-1}-\hat{\alpha} P_{t-1}\right)^{+}$ as separate regressors identifying $\lambda_{1}$ and $\lambda_{2}$.

The adjustment speed parameters, $\lambda_{1}$ and $\lambda_{2}$ and the parameter of asymmetric adjustment costs, $\delta$ of Eq. (4.1) and Eq. (4.2) are related. To link, the notion of asymmetric speeds of adjustment $\left(\lambda_{1}, \lambda_{2}\right)$ with the notion of asymmetry in adjustment costs $(\delta)$, we derive a piecewise closed form solution of (4.3) depending on the direction of the adjustment as follows. Linearizing (4.2) using a piecewise second order Taylor series expansion gives

$$
\operatorname{AAC}\left((1-B) Q_{t}\right) \equiv\left[\begin{array}{ll}
\gamma_{1}\left((1-B) Q_{t}\right)^{2} & \text { iff }(1-B) Q_{t}>0 \\
\gamma_{2}\left((1-B) Q_{t}\right)^{2} & \text { iff }(1-B) Q_{t}<0 \\
& 0 \text { otherwise }
\end{array}\right.
$$

where $\gamma_{1}$ and $\gamma_{2}$ are constant positive cost parameters of respectively rising and declining adjustment. Expression (4.7) implies that (see Pfann, 1996, page 326) ${ }^{3}$

$$
\begin{array}{ll}
\gamma_{1}>\gamma_{2} & \text { iff } \delta>0 \\
\gamma_{1}<\gamma_{2} & \text { iff } \delta<0 \\
\gamma_{1}=\gamma_{2} & \text { iff } \delta=0 .
\end{array} \quad \text { and }
$$

Thus, in order to obtain a closed form solution of the non-linear second order difference Eq. (4.3) the continuously differentiable asymmetric specification (4.2) has been approximated by a piecewise linear quadratic expansion. From the previous section the closed form solution for each piecewise linear-quadratic approximation is known. The two linearized necessary conditions are

$$
\begin{aligned}
E\left[(1-B) Q_{t+1} \mid \Omega_{t}\right]=\beta^{-1}(1-B) Q_{t}+\left(\beta \gamma_{1}\right)^{-1}\left(Q_{t}-Q_{t}^{*}\right), & \\
& \text { iff }(1-B) Q_{t}>0
\end{aligned}
$$

and

$$
\begin{aligned}
E\left[(1-B) Q_{t+1} \mid \Omega_{t}\right]=\beta^{-1}(1-B) Q_{t}+\left(\beta \gamma_{2}\right)^{-1}\left(Q_{t}-Q_{t}^{*}\right) & \\
& \text { iff }(1-B) Q_{t}<0 .
\end{aligned}
$$

When $Q_{t}-Q_{t}^{*}$ is negative, we expect $Q_{t}$ to rise in the next period.

\footnotetext{
${ }^{3}$ If $(1-B) Q_{t}>0, \gamma_{1}=\gamma+\delta^{2}$ and $\gamma_{2}=\gamma$, whereas if $(1-B) Q_{t}<0, \gamma_{1}=\gamma$ and $\gamma_{2}=\gamma+\delta^{2}$.
} 
Thus $(1-B) Q_{t}>0$ corresponds with $\left(Q_{t}-\alpha P_{t}\right)^{+}$and vice versa, i.e. when $Q_{t}$ $-Q_{t}^{*}$ is positive, we expect $Q_{t}$ to fall in the next period. So $(1-B) Q_{t}<0$ corresponds with $\left(Q_{t}-\alpha P_{t}\right)^{-}$. The closed form solution of (4.9) is therefore the NEC model (4.6). The relationships between the adjustment speed parameters $\left(\lambda_{1}, \lambda_{2}\right)$ of (4.6) and the parameters of the piecewise linearly approximated asymmetric adjustment costs model are as follows

$$
\begin{aligned}
& \lambda_{1}=\frac{1}{2}\left(1+\beta^{-1}+\left(\beta \gamma_{1}\right)^{-1}\right)-\frac{1}{2}\left(\left(1+\beta^{-1}+\left(\beta \gamma_{1}\right)^{-1}\right)^{2}-4 \beta^{-1}\right)^{1 / 2} \\
& \lambda_{2}=\frac{1}{2}\left(1+\beta^{-1}+\left(\beta \gamma_{2}\right)^{-1}\right)-\frac{1}{2}\left(\left(1+\beta^{-1}+\left(\beta \gamma_{2}\right)^{-1}\right)^{2}-4 \beta^{-1}\right)^{1 / 2}
\end{aligned}
$$

This completes the formal derivation of the relationship between asymmetric error correction models and asymmetric adjustment models.

\section{Non-linear error correction models and the implications of having trends in the mean}

In the case of $Q_{t}$ and $P_{t}$ having trends in the means the NEC representation specified in terms of $P_{t}$ and $Q_{t}$ according to Eq. (2.10) is as follows

$$
\begin{aligned}
\phi_{\alpha}(B)(1-B) Q_{t} & +\theta_{\alpha}(1-B) P_{t}=\phi_{\alpha}(B)(1-B) \mu_{q t}-\theta_{\alpha}(B)(1-B) \mu_{p t} \\
& +\Gamma_{1}\left(\mu_{q t-1}-\alpha \mu_{p t-1}\right)-\Gamma_{1}\left(Q_{t-1}-\alpha P_{t-1}\right) \\
& -g_{\alpha}\left(-\mu_{q t-1}+\alpha \mu_{p t-1}+Q_{t-1}-\alpha P_{t-1}\right)+\epsilon_{\alpha t} .
\end{aligned}
$$

However, Eq. (5.1) is usually written as

$$
\begin{aligned}
& \phi_{\alpha}(B)(1-B) Q_{t}+\theta_{\alpha}(B)(1-B) P_{t} \\
& \quad=C_{1}-\Gamma_{1}\left(Q_{t-1}-\alpha P_{t-1}\right)-g_{\alpha}\left(Q_{t-1}-\alpha P_{t-1}\right)+\epsilon_{\alpha t} .
\end{aligned}
$$

For (5.2) to be a well specified model several conditions need to be satisfied. Differencing once should be a good detrending procedure for the means so that $(1-B) \mu_{q t}$ and $(1-B) \mu_{p t}$ are not trending. The trends in mean of $Q_{t}$ and $P_{t}$ should be proportional (co-trending in mean), such that $\mu_{q t}-\alpha \mu_{p t}$ is no longer trending. Notice also that the asymmetric terms of $g_{\alpha}($.$) are forced to satisfy also$ the requirements as well. The cointegrating vector $(1,-\alpha)$ is also the vector that is making the trending variables co-trending.

To obtain the structural counterpart of the asymmetric error correction model with $P_{t}$ and $Q_{t}$ having trends in the means we have to redefine some of the characterizations presented in Section 4. 
The error correction components now become

$$
\left(Q_{t}-\alpha P_{t}\right)^{+}=\left[\begin{array}{cc}
\left(Q_{t}-\alpha P_{t}\right)-\left(\mu_{q t}-\alpha \mu_{p t}\right) & \text { iff }(1-B)\left(\left(Q_{t}-\mu_{q t}\right)\right. \\
\left.-\alpha\left(P_{t}-\mu_{p t}\right)\right)>0 \\
0 \text { otherwise }
\end{array}\right.
$$

and

$$
\left(Q_{t}-\alpha P_{t}\right)^{-}=\left[\begin{array}{ll}
\alpha_{1}\left[(1-B)\left(Q_{t}-\mu_{q t}\right)\right]^{2} & \text { iff }(1-B)\left(Q_{t}-\mu_{q t}\right)>0 \\
\alpha_{2}\left[(1-B)\left(Q_{t}-\mu_{q t}\right)\right]^{2} & \text { iff }(1-B)\left(Q_{t}-\mu_{q t}\right)<0 \\
& 0 \text { otherwise. }
\end{array}\right.
$$

After these redefinitions Eq. (4.5) and Eq. (4.6) and Eqs. (4.8),(4.9a),(4.9b), (4.10a),(4.10b) applies.

To clarify the specification error when trends in the means are not correctly accounted for, a simple example is given in Fig. 1a,b. Here Eq. (4.4a) and Eq. (4.4b) are no longer true, because the area $\mathrm{A}^{\prime}$, that corresponds to $(1-B)\left(Q_{t}-\alpha P_{t}\right)=$ $(1-B) u_{t}$ is positive and $Q_{t}-\alpha P_{t}=u_{t}$ is positive as well. However, in area A, $(1-B)\left(Q_{t}-\alpha P_{t}\right)$ is negative, whereas $Q_{t}-\alpha P_{t}$ is positive. The same argument holds for the condition of Eq. (4.2) which is related to the areas $\mathrm{B}^{\prime}$ and B of Fig. 1a and the corresponding area of Fig. $1 \mathrm{~b}$.

Moreover, asymmetries may occur between situations where the growth rate of the observed decision variable $\left(Q_{t}\right)$ exceeds the growth rate of the target $Q_{t}^{*}$ on the one hand and situations where the growth rate of $Q_{t}$ is lower than the growth rate of $Q_{t}^{*}$ on the other hand. This asymmetry may even be observed during periods where the decision variable is above the target (see Fig. 1a,b). Similarly, we can account for asymmetry in growth rates between areas B and B'.

In order to implement the notion of trending asymmetry in an error correction framework, the adjustment towards the equilibrium should be a correspondence, instead of a function (see Fig. 2b) We are currently investigating the implications of trending asymmetries in dynamic time series models. This analysis, however, is considered to be beyond the scope of this article.

\section{Type of asymmetries}

In this section we consider more general forms of the non-linear function $g_{\alpha}($. that could be empirically interesting in the still young literature on non-linear error corrections and asymmetric adjustments. All the dynamic representations are in deviations from the mean, $\bar{Q}_{t}=Q_{t}-\mu_{q t}$ and $\bar{P}_{t}=P_{t}-\mu_{p t}$.

First, we consider a piecewise linear adjustment type of function (see Fig. 2c) 


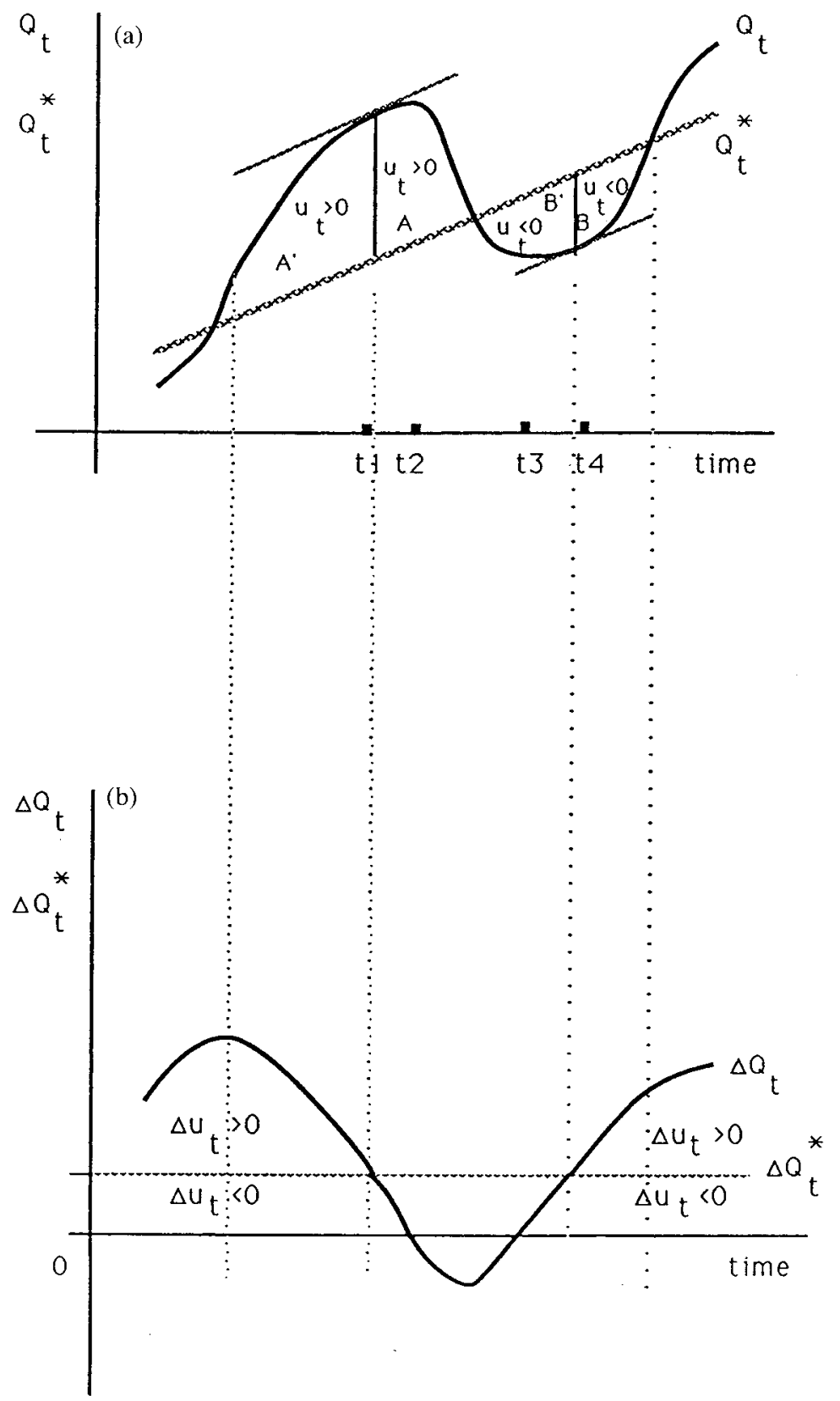

Fig. 1. Non-linear error correction with trend in mean. 


$$
\begin{aligned}
\Gamma_{1}\left(\bar{Q}_{t}-\alpha \bar{P}_{t}\right)+g_{\alpha}\left(\bar{Q}_{t}-\alpha P_{t}\right)=\mu_{1} D_{1 t}\left(\bar{Q}_{t}-\alpha \bar{P}_{t}\right) \\
-\mu_{2} D_{2 t}\left(\bar{Q}_{t}-\alpha \bar{P}_{t}\right)-\mu_{3} D_{3 t}\left(\bar{Q}_{t}-\alpha \bar{P}_{t}\right), \\
D_{1 t}=\left[\begin{array}{ll}
1 & \text { iff } \bar{Q}_{t}-\alpha \bar{P}_{t} \leq C^{-} \\
0 & \text { otherwise }
\end{array}\right. \\
D_{2 t}=\left[\begin{array}{ll}
1 & \text { iff } C-\leq \bar{Q}_{t}-\alpha \bar{P}_{t} \leq C^{+} \\
0 & \text { otherwise }
\end{array}\right. \\
D_{3 t}=\left[\begin{array}{ll}
1 & \text { iff } \bar{Q}_{t}-\alpha \bar{P}_{t} \geq C^{+} \\
0 & \text { iff } \bar{Q}_{t}-\alpha \bar{P}_{t} \geq C^{-} .
\end{array}\right.
\end{aligned}
$$

From Fig. $2 \mathrm{c}$ it is clear that the equilibrium is unique, although the adjustment is slower in the small interval $\left(C^{-}, C^{+}\right)$around the equilibrium and faster out of this range. This type of asymmetric adjustment represents a generalization of the type of asymmetries analyzed by Granger and Lee (1989) (see Fig. 2a). Their model is obtained when $D_{2 t}=0$ and $C^{-}=C^{+}=0$. However, to impose uniqueness of the equilibrium around the 0 point may be too restrictive in general. If, in the interval $\left(C^{-}, C^{+}\right)$close to the equilibrium there is no adjustment (see Fig. 3D), a continuum of equilibria exists. Particular cases of interest that are nested in this formulation are obtained if $C^{-}=0$ and $C^{+}>0$, or if $C^{-}<0$ and $C^{+}=0$. In fact the model consider by Hendry and Ericsson (1991) is observationally equivalent to a piece wise linear adjustment with $C^{-}=0$ and $C^{+}=0.2$

Next, we consider a second type of function, namely the more general cubic polynomials (see Fig. 2e,f)

$$
\begin{aligned}
& \Gamma_{1}\left(\bar{Q}_{t}-\alpha \bar{P}_{t}\right)+g_{\alpha}\left(\bar{Q}_{t}-\alpha \bar{P}_{t}\right) \\
& \quad=\mu_{1}\left(\bar{Q}_{t}-\alpha \bar{P}_{t}\right)+\mu_{2}\left(\bar{Q}_{t}-\alpha \bar{P}_{t}\right)^{2}+\mu_{3 t}\left(\bar{Q}_{t}-\alpha \bar{P}_{t}\right)^{3}
\end{aligned}
$$

where $\mu_{3 t}$ is time dependent for very large values of $\bar{Q}_{t}-\alpha \bar{P}_{t}$ in order to guarantee the asymptotic stability conditions (see Escribano, 1986) or the near epoch dependence conditions (NED) (see Escribano, 1996). This cubic polynomial has several advantages over general piecewise linear asymmetries. It determines endogenously the range of equilibria $\left(C^{-}, C^{+}\right)$, therefore allowing the adjustment to by asymmetric and non-linear in each regime. Fig. $2 \mathrm{f}$ represents the adjustment mechanism observed for UK money demand during the period 1878-1970 (see Escribano, 1996). Eq. (6.2) has the nice property that the adjustment is faster when the distance between the decision variable and the target becomes larger and, in general, it can be considered a simple approximation to more general unknown adjustment mechanisms, which could be estimated by non-parametric techniques (smoothing splines). 

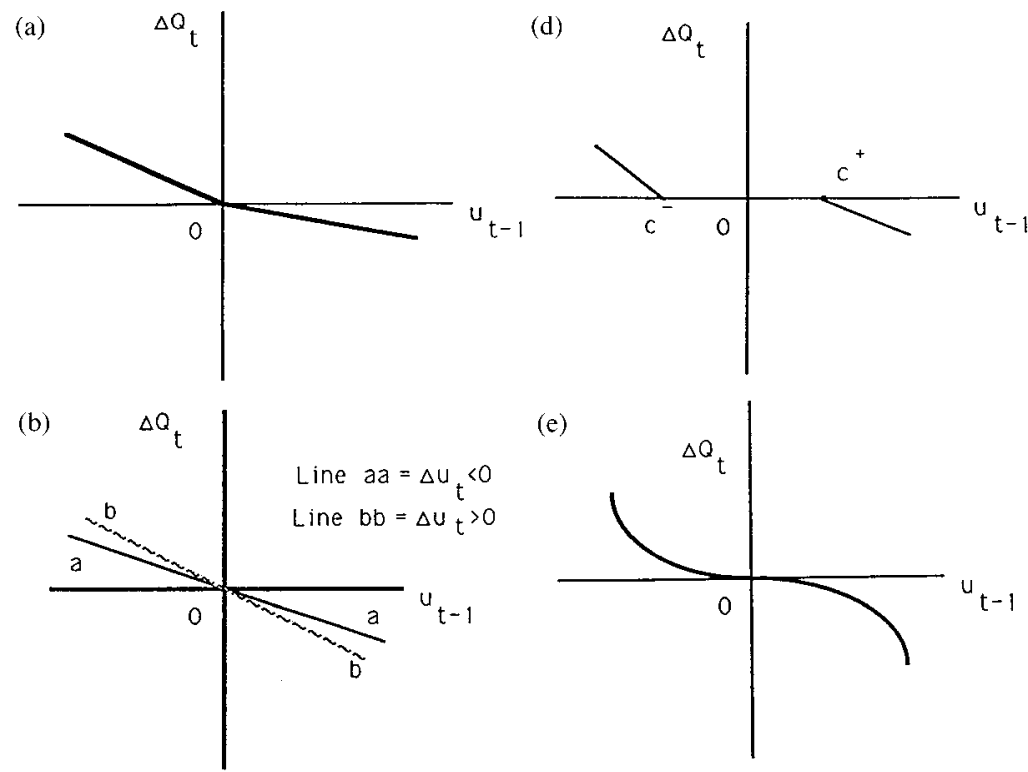

(e)
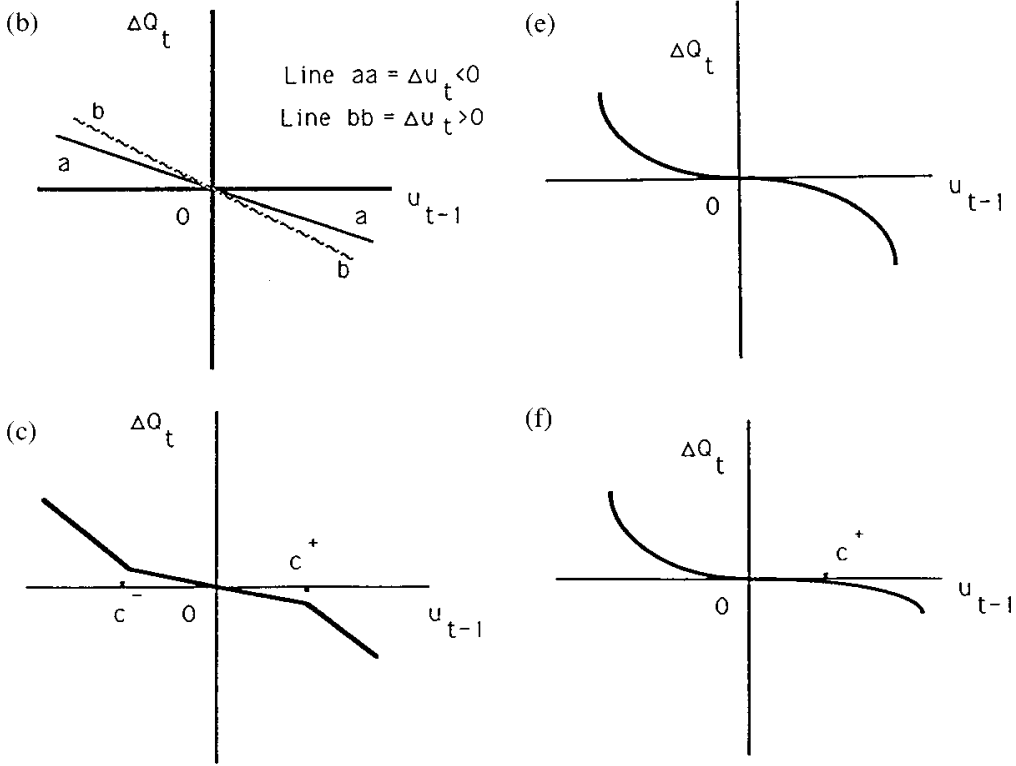

Fig. 2. Several types of asymmetries.

Another interesting parametric family is the rational polynomials introduced in Escribano (1996).

$$
\begin{aligned}
\Gamma_{1}\left(\bar{Q}_{t}-\alpha \bar{P}_{t}\right)+g_{\alpha}\left(\bar{Q}_{t}-\alpha \bar{P}_{t}\right) \\
\quad=\frac{\mu_{1}\left(\bar{Q}_{t}-\alpha \bar{P}_{t}\right)+\mu_{2}\left(\bar{Q}_{t}-\alpha \bar{P}_{t}\right)^{2}+\ldots+\mu_{L}\left(\bar{Q}_{t}-\alpha \bar{P}_{t}\right)^{L}}{\theta_{1}\left(\bar{Q}_{t}-\alpha \bar{P}_{t}\right)+\theta_{2}\left(\bar{Q}_{t}-\alpha \bar{P}_{t}\right)^{2}+\ldots+\theta_{M}\left(\bar{Q}_{t}-\alpha \bar{P}_{t}\right)^{M}},
\end{aligned}
$$

By the convergence results of Padé approximants, this class of models can approximate arbitrarily well any analytic function while satisfying, at the same time, 
the required stability conditions for near epoch dependence (NED) or asymptotic uncorrelation. For example, Fig. 2e,f could have been generated by this class of models by choosing polynomials of degree three in the numerator and two in the denominator, $L=3$ and $M=2$.

\section{An empirical application}

The asymmetric adjustment error correction approach may prove useful in structurally analyzing any economic time series that is assumed to be endogenously generated by the optimizing behavior of (representative) agents. Examples are investment: Gould (1968), consumption: Hall (1978) and so forth. The empirical application presented in this section will be limited to the theory and practice of dynamic labor demand and is founded on the research described in Pfann (1990).

The following notations will be used. $L_{t}=$ the number of white collar workers employed in the UK manufacturing sector at time $t ; W_{t}=$ the real UK manufacturing sector white collar wage costs at time $t ; K_{t}=$ the UK manufacturing sector capital stock at time $t$. The annual UK data run from 1955 to 1986 (see Appendix 1 for the sources and the definitions).

The characteristics of the series are as follows (see Appendix 2)

1. $L_{t}, K_{t}$ and $W_{t}$ have a unit root.

2. $L_{t}, K_{t}$ and $W_{t}$ have one cointegrating vector.

In this example the decision variable is white collar employment and the set of forcing variables consists of real white collar wage costs and capital. We have chosen white collar workers for the empirical illustration, because it is well-known from previous research that white collar employment is a quasi-fixed production factor that bears asymmetric adjustment costs when being changed. In correspondence with the preceding section, the following relation holds

$$
Q_{t}=L_{t}, \quad \text { and } \quad P_{t}=\left\{K_{t}, W_{t}\right\} .
$$

The equilibrium errors from the cointegration relationship are as follows

$$
\begin{aligned}
& \hat{u}_{t}=L_{t}+\underset{(4.41)}{5.35}+\underset{(8.20)}{0.28} \mathrm{OC} 74+\underset{(9.32)}{1.89} W_{t}-\underset{(10.66)}{2.59} K_{t} \\
& R^{2}=0.49 \quad \sigma=0.056 \quad \mathrm{ADF}=-4.56
\end{aligned}
$$

In all equations absolute $t$-values are given within parentheses. OC74 is a step-dummy for the oil crisis of fall 1973 being one from 1974 on. In addition to the correctly specified test of the cointegrating vectors of the system (see Phillips, 1991), as presented in Appendix 2, we also present the Augmented Dickey Fuller statistic (ADF) in (7.1).

Judging from Table IIb of Phillips and Ouliaris (1990) the hypothesis of no 


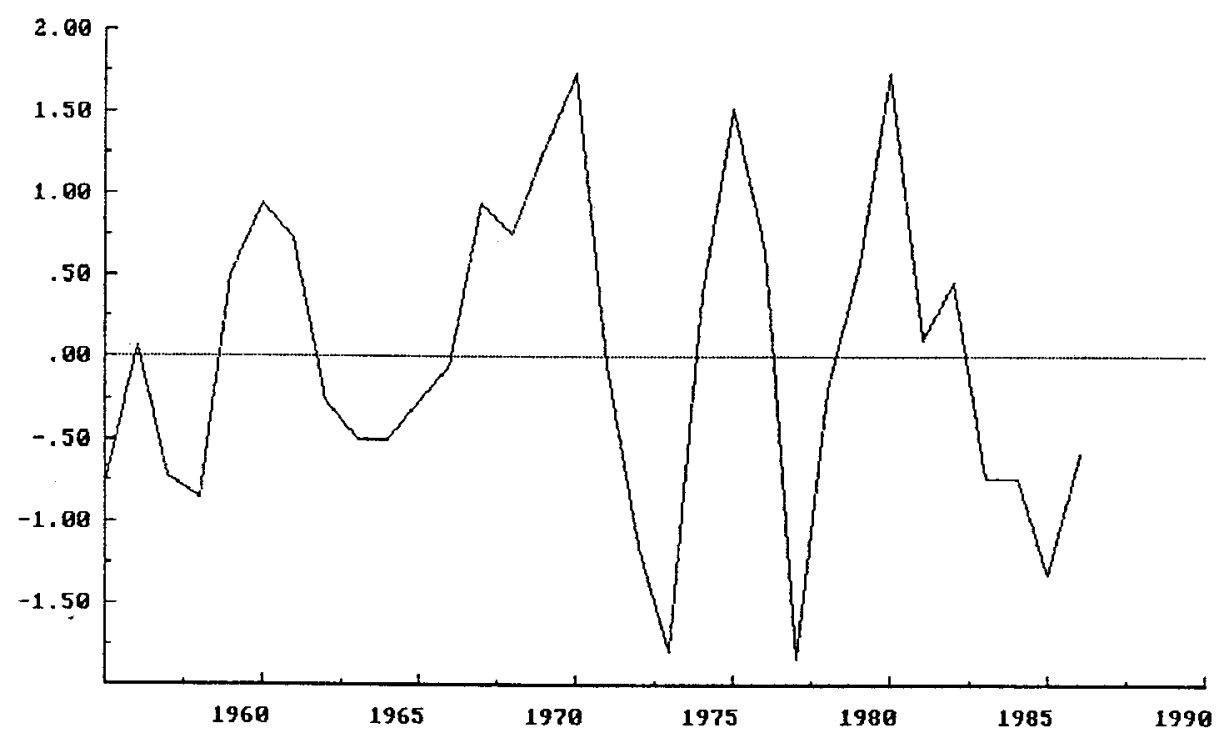

Fig. 3. Longrun errors of $\mathrm{L}, \mathrm{K}$ and $\mathrm{W}$, Cointegration relation.

cointegration is rejected in Eq. (7.1). Leaving out OC74 reduced the Augmented Dickey Fuller statistic (ADF) to - 1.49. Consequently, structural breaks may blur cointegration relations if they are not adequately dealt with (see also Perron, 1989; Palm and Pfann, 1991).

Fig. 3 shows $\hat{u}_{t}$ of Eq. (7.1), where the horizontal line plays the role of the longrun equilibrium between $L_{t}, K_{t}$ and $W_{t}$.

The estimated linear error correction model (3.12) is as follows

$$
(1-B) \hat{L}_{t}=-\underset{(4.16)}{0.0006}-\underset{(0.32)}{0.007}(1-B) W_{t-1}+\underset{(4.36)}{2.28(1-B) K_{t-1}}-\underset{(1.90)}{0.24} \hat{u}_{t-1}
$$

Sample $=1957-1986$

$$
R^{2}=0.46 \quad \sigma=0.033 \quad \chi_{\mathrm{AR}}^{2}(2)=0.70 \quad \chi_{\mathrm{NORM}}^{2}(2)=1.85 \quad \chi_{\mathrm{ARCH}}^{2}(2)=0.78
$$

The reported statistics are the residual based Ljung-Box test for residual autocorrelation $\left(\chi_{\mathrm{AR}}^{2}\right)$, the residual based normality test $\left(\chi_{\mathrm{NORM}}^{2}\right)$ and the residual based ARCH test $\left(\chi_{\mathrm{ARCH}}^{2}\right)$. All tests have two degrees of freedom. None of the tests are significant. Thus the model would be an acceptable econometric model. Next, we report the estimated asymmetric error correction model (4.5)

$$
(1-B) \hat{L}_{t}=-\underset{(3.37)}{0.06}-\underset{(0.25)}{0.05}(1-B) W_{t-1}+\underset{(4.13)}{2.17(1-B) K_{t-1}}
$$




$$
-\underset{(2.19)}{0.42} \hat{u}_{t-1}^{+}-\underset{(0.41)}{0.07} \hat{u}_{t-1}^{-}
$$

Sample $=1957-1986$

$$
R^{2}=0.49 \quad \sigma=0.032 \quad \chi_{\mathrm{AR}}^{2}(2)=0.24 \quad \chi_{\mathrm{NORM}}^{2}(2)=1.95 \quad \chi_{\mathrm{ARCH}}^{2}(2)=0.51
$$

The estimated error correction parameters in (7.3) provide us with useful additional information with respect to the asymmetry between underequilibrium adjustment and overequilibrium adjustment towards the longrun cointegration relation. The finding that $\hat{u}_{t-1}^{-}$and $\hat{u}_{t-1}^{+}$both have negative signs is in line with the expected error corrections for procyclical variables. The models are not statistically distinct: the $F$-statistic testing the statistical significance of the included asymmetry (7.3) vs. the linear symmetric model (7.2) yields $F_{1,25}=1.58$. However, the adjustment speed towards a higher target level of white collar employment $\left(1-\gamma_{1}=\right.$ $0.42)$ exceeds the adjustment speed towards a lower target level $\left(1-\gamma_{2}=0.07\right)$. The characteristic roots $\lambda_{1}=0.58$ and $\lambda_{2}=0.93$ lie within the unit circle. Using (5.9a) and (5.9b) the piecewise linear asymmetric adjustment cost parameters yield $\gamma_{1}=3.07$ and $\gamma_{2}=114.04$, assuming $\tau=0.95$. Thus, we find that $\gamma_{1}<\gamma_{2}$, implying $\delta<0$ [see (4.6)], which is in accordance with the related findings on asymmetry for white collar workers (see Pfann and Palm, 1993).

\section{Conclusions}

In this article we showed that non-linear error correction mechanisms that are found to exist in macroeconomic time series data may be endogenously generated resulting from the optimizing behavior of (representative) agents that face asymmetric costs of adjustment. The rationale for asymmetric costs is equivalent to the notion of non-linear error correction mechanisms: the adjustment path to a higher target level should not necessarily be symmetric with the adjustment path to a lower target level. Several new types of asymmetries are discussed. We saw how by extending the piecewise linear error correction of Granger and Lee (1989) to more general adjustments we allow for multiple equilibria. Parametric functions like cubic polynomials or rational polynomials can endogenously generate all the proposed desired elements, asymmetries and multiple equilibria. Furthermore, we explained how trends should be included in the non-linear error correction model. In a numerical example we estimated the adjustment speeds in different phases of the economic cycle for UK manufacturing white collar workers, finding that white collar workers are more easily hired in times of economic growth than fired in times of economic recessions.

\section{Acknowledgements}

We are grateful to an anonymous referee and to Juan Dolado, Clive Granger and Franz Palm for constructive comments. The stimulating research environments of the Universite Catholique de Louvain-la-Neuve, Institute for Empirical 
Macroeconomics in Minneapolis, University of California, Los Angeles (conference on Non-linear Dynamics) and University of Amsterdam ((EC)2), also influenced the article's contents substantially. Alvaro Escribano acknowledges financial support from the SPES program of the European Commission and the Spanish DGICYT PB95-0298 and Gerard Pfann from the European Commission SPES programme and the Royal Dutch Academy of Arts and Sciences (KNAW).

\section{Appendix 1}

\section{Sources of the annual UK manufacturing data}

The base year of all prices and indices is 1980. Sample: 1955-1986. The following main data sources were used:

BB: $\quad$ Blue Book

DEG: Department of Employment Gazette

ETAS: Economic Trend Annual Supplement

HABLS: Historical Abstract of British Labour Statistics

MM: Mendis L. and J. Muellbauer (1984), British Manufacturing Productivity 1955-1983: Measurement Problems, Oil Shocks and Thatcher Effects, CEPR Discussion Paper No. 34.

The variables are defined as follows:

L: The natural log of the total numbers of employees in UK manufacturing, have been obtained from ETAS.

W: The natural log of the real weekly earnings index have been obtained by deflating gross weekly earnings of manual and non-manual workers (pre-1970 data: HABLS; from 1970 on data: New Earnings Survey in DEG) by Py.

$\mathrm{K}$ : The natural log of the gross capital stock at constant prices $(K)$ have been obtained from BB for data from 1963 and from MM for pre-1963 data.

\section{Appendix 2}

\section{Unit roots and cointegration}

Unit root tests

Model: $(1-B) V_{t}=a^{\prime} X_{t}+\alpha_{1} V_{t-1}+\alpha_{2}(1-B) V_{t-1}+\epsilon_{t}$

$V_{t} \in\left\{L_{t}, K_{t}, W_{t}\right\}$

$X_{t}+(\text { CONST, OC74 })^{\mathrm{a}}$

$H_{0}: \alpha_{1}=0$

Sample: $1957-1986$

\begin{tabular}{lrrr}
\hline & \multicolumn{1}{c}{$L_{t}$} & \multicolumn{1}{c}{$K_{t}$} & \multicolumn{1}{c}{$W_{t}$} \\
\hline Fuller's $\hat{\tau}_{\tau}$ & -1.37 & -2.53 & -0.92 \\
Adjusted $R^{2}$ & 0.33 & 0.85 & 0.50 \\
DW-statistic & 2.01 & 1.42 & 1.96 \\
\hline
\end{tabular}


${ }^{\mathrm{a}} \mathrm{OC74}$ is a step-dummy equal to 1 in 1974 and zero elsewhere.

According to Fullers's $\hat{\tau}_{\tau}$ statistic (Fuller, 1976, Table 8.5.2), we do not reject the hypothesis that the univariate time series have a unit root. Also, if we take account of the fact the one dummy variable (OC74) is included in the model and therefore use the distribution given by Perron (1989), we reach the same conclusion.

\section{Johansen's cointegration tests}

Explanatory variable: $L_{t}$ Forcing variables: $W_{t}, K_{t}$ Sample: $1957-1986$

$\mathrm{CV}$, number of cointegration vectors.

\begin{tabular}{llr}
\hline & & $H_{0}: r$ \\
\hline$C V=1$ & $(r=0)$ & 33.46 \\
$C V=2$ & $(r=1)$ & 4.01 \\
\hline
\end{tabular}

The critical values for a three variate cointegration system are given in Table 1 of Johansen (1988): 23.8 and 26.1 for $5 \%$ and $2.5 \%$ significant levels respectively. We find that the hypothesis of no cointegration is rejected in favor of one cointegration vector.

\section{References}

Banerjee, A., Dolado, J., Galbraith, J.W., Hendry, D.F., 1993. Co-integration, Error-Correction and the Econometric Analysis of Non-Stationary Data. Oxford University Press, Oxford, UK.

Bewley, R.A., 1979. The Direct Estimation of the Equilibrium Response in a Linear Model. Econ. Lett. 3.

Engle, R.F., Hendry, D.F., Richard, J.-F., 1983. Exogeneity. Econometrica 51, 277-304.

Engle, R.F., Granger, C.W.J., 1987. Cointegration and error correction: representation, estimation and testing. Econometrica 55, 251-276.

Escribano, A., 1986. Identification and Modeling of Economic Relationships in a Growing Economy. Ph.D. Thesis, University of California, San Diego.

Escribano, A., 1987. Error Correction Systems: Nonlinear Adjustments to Linear Long-run Relationships. CORE Discussion Paper 8730, University of Louvain.

Escribano, A., 1996. Nonlinear Error Correction: The Case of Money Demand in the U.K. (1878-1970). W.P. 96-55-24 Universidad Carlos III de Madrid.

Escribano, A., Mira, S., 1997. Nonlinear Error Correction Models. W.P. 9726-13 Universidad Carlos III de Madrid.

Fuller, W.A., 1976. Introduction to Statistical Time Series. John Wiley, New York.

Gould, J.P., 1968. Adjustment costs in the theory of investment of the firm. Rev. Econ. Stud. 35, 47-56.

Granger, C.W.J., Lee, T.-H., 1989. Investigation of production, sales and inventory relationships using multicointegration and non-symmetric error correction models. J. Appl. Econometr. 4, 145-159.

Hall, R.E., 1978. Stochastic implications of the life-cycle-permanent income hypothesis: theory and evidence. J. Pol. Econ. 86, 461-481.

Hamilton, J.D., 1989. A new approach to the economic analysis of nonstationary time series and the business cycle. Econometrica 57, 357-384.

Hansen, L.P., Sargent, T.J., 1980. Formulating and estimating dynamic linear rational expectations models. In: Lucas, R.E. Jr., Sargent, T.J. (Eds.), Rational Expectations and Economic Practice. University Minnesota Press, Minneapolis.

Hendry, D.F., Ericsson, N.R., 1991. An econometric analysis of UK money demand in monetary trends 
in the United States and the United Kingdom by Milton Friedman and Anna J. Schwarz. Am. Econ. Rev. 81.1, 8-38.

Hylleberg, S., Mizon, G.E., 1989. Cointegration and error correction mechanisms. Econ. J. (Supplement) 99, 113-125.

Johansen, S., 1988. Statistical analysis of cointegration vectors. J. Econ. Dyn. Control 12, 231-254.

Nickell, S.J., 1985. Error correction, partial adjustment and all that: An expository note. Oxford Bull. Econ. Stat. 47, 119-129.

Novales, A., 1990. Solving nonlinear rational expectations models: A stochastic equilibrium model of interest rates. Econometrica 58, 93-112.

Palm, F.C., Pfann, G.A., 1991. Interrelation, Structural Changes, and Cointegration in a Model for Manufacturing Demand in the Netherlands. Recherches Economiques de Louvain, p. 57.

Palm, F.C., Pfann, G.A., 1997. The sources of asymmetry in dynamic factor demands. J. Econometr., forthcoming.

Perron, P., 1989. The great crash, the oil price shocks, and the unit root hypothesis. Econometrica 57, $1361-1402$.

Pfann, G.A., 1990. Dynamic Modelling of Stochastic Demand for Manufacturing Employment. Lecture Notes in Economics and Mathematical Systems 349, Springer-Verlag, Berlin.

Pfann, G.A., 1996. Factor demand models with nonlinear shortrun fluctuations. J. Econ. Dyn. Control 20, 315-331.

Pfann, G.A., Palm, F.C., 1993. Asymmetric adjustment costs in labour demand models. Rev. Econ. Stud. 60, 397-412.

Pfann, G.A., Verspagen, B., 1989. The structure of adjustment costs for labour in the Dutch manufacturing sector. Econ. Lett. 29, 365-371.

Phillips, P.C.B., Ouliaris, S., 1990. Asymptotic properties of residual based tests for cointegration. Econometrica 58, 165-193.

Phillips, P.C.B., 1991. Optimal inference in cointegrated systems. Econometrica 59, 283-306.

Stock, J., 1987. Asymptotic properties of least squares estimators of cointegrating vectors. Econometrica $55,1035-1056$.

Wickens, M.R., Breusch, T.S., 1988. Dynamic specification, the long run and the estimators of transformed regression models. Econ. J. Conf. Papers 99, 189-205. 